\section{(2)}

Author affiliations and support information (if applicable) appear at the end of this article.

Published at jco.org on January 25, 2018. C.U. and A.G. contributed equally to this work.

Clinical trial information: NCT02289014.

Corresponding author: Viviane Hess, MD, University Hospital Basel, Medical Oncology, Petersgraben 4, CH-4031 Basel, Switzerland; e-mail: viviane.hess@ usb.ch.

(C) 2018 by American Society of Clinical Oncology. Creative Commons Attribution NonCommercial No Derivatives 4.0 License. (c) (i) (5)

0732-183X/18/3608w-780w/\$20.00

\section{ASSOCIATED CONTENT}

(2) Appendix

DOI: https://doi.org/10.1200/JCO 2017.74.8491

Data Supplement

DOI: https://doi.org/10.1200/JCO. 2017.74.8491

DOI: https://doi.org/10.1200/JCO.2017. 74.8491

\title{
Web-Based Stress Management for Newly Diagnosed Patients With Cancer (STREAM): A Randomized, Wait-List Controlled Intervention Study
}

Corinne Urech, Astrid Grossert, Judith Alder, Sandra Scherer, Barbara Handschin, Benjamin Kasenda, Borislava Borislavova, Sven Degen, Jennifer Erb, Alexandra Faessler, Laura Gattlen, Sarah Schibli, Celine Werndli, Jens Gaab, Thomas Berger, Thomas Zumbrunn, and Viviane Hess

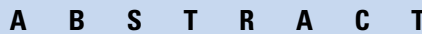

\section{Purpose}

Being diagnosed with cancer causes major psychological distress; however, a majority of patients lack psychological support during this critical period. Internet interventions help patients overcome many barriers to seeking face-to-face support and may thus close this gap. We assessed feasibility and efficacy of Web-based stress management (STREAM [Stress-Aktiv-Mindern]) for newly diagnosed patients with cancer.

\section{Patients and Methods}

In a randomized controlled trial, patients with cancer who had started first-line treatment within the previous 12 weeks were randomly assigned to a therapist-guided Web-based intervention or a waitlist (control), stratified according to distress level ( $\geq 5 v<5$ on scale of 0 to 10). Primary efficacy end point was quality of life after the intervention (Functional Assessment of Chronic IIIness Therapy-Fatigue). Secondary end points included distress (Distress Thermometer) and anxiety or depression (Hospital Anxiety and Depression Scale). Treatment effect was assessed with analyses of covariance, adjusted for baseline distress.

\section{Results}

A total of 222 of 229 screened patients applied online for participation. Between September 2014 and November 2016, 129 newly diagnosed patients with cancer, including 92 women treated for breast cancer, were randomly assigned to the intervention $(n=65)$ or control $(n=64)$ group. Adherence was good, with $80.0 \%$ of patients using $\geq$ six of eight modules. Psychologists spent 13.3 minutes per week (interquartile range, 9.5-17.9 minutes per week) per patient for online guidance. After the intervention, quality of life was significantly higher (Functional Assessment of Chronic Illness Therapy-Fatigue: mean, 8.59 points; $95 \% \mathrm{Cl}, 2.45$ to 14.73 points; $P=.007$ ) and distress significantly lower (Distress Thermometer: mean, $-0.85 ; 95 \% \mathrm{Cl},-1.60$ to $-0.10 ; P=.03$ ) in the intervention group as compared with the control. Changes in anxiety or depression were not significant in the intention-to-treat population (Hospital Anxiety and Depression Scale: mean, -1.28; $95 \% \mathrm{Cl},-3.02$ to $0.45 ; P=.15)$. Quality of life increased in the control group with the delayed intervention.

\section{Conclusion}

The Web-based stress management program STREAM is feasible and effective in improving quality of life.

J Clin Oncol 36:780-788. (c) 2018 by American Society of Clinical Oncology. Creative Commons Attribution Non-Commercial No Derivatives 4.0 License: https://creativecommons.org/licenses/by-nc-nd/4.0/

\section{INTRODUCTION}

Diagnosis of cancer elicits high levels of distress in a majority of patients, ${ }^{1}$ which is associated with decreased quality of life as well as diminished treatment tolerance $^{2,3}$ and potentially worse disease course.,
Psychosocial support for patients with cancer is effective in alleviating distress and improving quality of life, including fatigue, the most common complaint of patients with cancer. ${ }^{2,6}$ However, a majority of newly diagnosed patients with cancer lack psychosocial support because of constraints on the part of both providers and patients. ${ }^{6,7}$ Use of the Internet, which 
has become an integral part of our lives, has the potential to change this. At least $70 \%$ of patients with cancer use the Internet as a source of information shortly after diagnosis, ${ }^{8}$ making it a powerful platform for reaching these patients. Recent approaches to integrating the Internet into patient care range from patient forums to information sites and even therapeutic games. ${ }^{9,10}$ Internet programs based on cognitive behavioral techniques with patient guidance via regular online contact with a health care professional (ie, therapist-guided programs or guided self-help) have emerged as particularly effective options. For a range of psychological disorders, including anxiety disorders and depression in those without cancer, ${ }^{11,12}$ therapist-guided online interventions seem similarly effective as face-to-face interventions. ${ }^{13}$ The success of Web-based guided selfhelp in psychological disorders ${ }^{9,13}$ coupled with the need to further improve access to psychosocial support for patients with cancer, especially outside of inner cities with large cancer centers, ${ }^{6}$ has boosted interest in online interventions in oncology. Numerous piloted and ongoing trials in patients with cancer seek to define suitable indications, formats, and settings. ${ }^{14}$ The few larger published randomized controlled trials ${ }^{15-17}$ show encouraging results, with improvement in a number of relevant psychosocial domains, including coping with cancer, ${ }^{15}$ sexual functioning, ${ }^{16}$ and distress ${ }^{17}$ in breast cancer survivors.

We designed the STREAM (Stress-Aktiv-Mindern) intervention specifically for the particularly vulnerable period immediately after first diagnosis of cancer. ${ }^{18}$ The rationale behind this early intervention was three-fold. First, distress in patients with cancer peaks shortly after diagnosis, ${ }^{18}$ irrespective of cancer type. Second, the time after diagnosis is busy with appointments for diagnostics and treatment. Therefore, the self-management of time and location allowed by Webbased interventions ${ }^{9}$ might be of particular value. Third, successful early psychosocial interventions have shown potential to affect disease course beyond psychosocial outcomes. ${ }^{19}$ We assessed feasibility and efficacy of our therapist-guided Web-based stress management program STREAM for newly diagnosed patients with cancer receiving first-line treatment.

\section{PATIENTS AND METHODS}

Details are provided in the Appendix (online only) and the published protocol. ${ }^{20}$ We included adult patients (age $\geq 18$ years) with newly diagnosed cancer who started first-line treatment (either systemic treatment, including chemotherapy, hormonal treatment, or targeted therapy, or radiotherapy) no longer than 12 weeks before study registration. Patients were required to provide written informed consent, read and write in German, and have Internet access as well as basic computer skills. The ethics committee approved the study (EKNZ339/13). Patients were recruited online via the STREAM Web site of STREAM. We randomly assigned eligible patients at a ratio of one to one using blocked randomization with randomly selected block sizes to an intervention group or a wait-list control group (Fig 1). Patients were stratified according to baseline distress using an internationally accepted cutoff of $\geq 5$ points on the 10-point visual analog scale (VAS) of the Distress Thermometer (DT). ${ }^{21}$

\section{Intervention}

We developed the Web-based intervention STREAM $^{20}$ based on established stress management intervention manuals ${ }^{22}$ that incorporate cognitive behavioral- and mindfulness-based stress reduction techniques, which we adapted to the Web context. STREAM consists of eight modules (Appendix Table A1, online only), which can be completed in 60 to 90 minutes each. Daily use of downloadable audio files with relaxation and guided-imagery exercises was encouraged. Participants were asked to complete one module per week. Our therapists provided weekly written feedback via integrated secured e-mail.

Patients in the control group underwent their cancer treatment locally as planned and were recontacted by the study team 8 weeks after random assignment (T2; Fig 1). After T2 assessments, they received access to the online program. For patients in both groups, cancer treatment was determined locally, and supportive care according to local standards may also have included face-to-face psychosocial support and psychotropic drugs.

\section{Assessments}

Assessments were conducted electronically directly within the Webbased program via the open source application LimeSurvey at baseline (T1) and after the intervention or waiting period (control group), respectively (T2). In addition, 2-month follow-up (T3) was performed in both groups.

\section{Efficacy End Points}

Primary end point was quality of life at T2, assessed using the validated German version of the Functional Assessment of Chronic Illness Therapy-Fatigue (FACIT-F) questionnaire. ${ }^{23}$ Minimal clinically meaningful differences are not well defined but have previously been set between 7 and 9 points, both as intraindividual changes and differences in groups. ${ }^{19,24}$

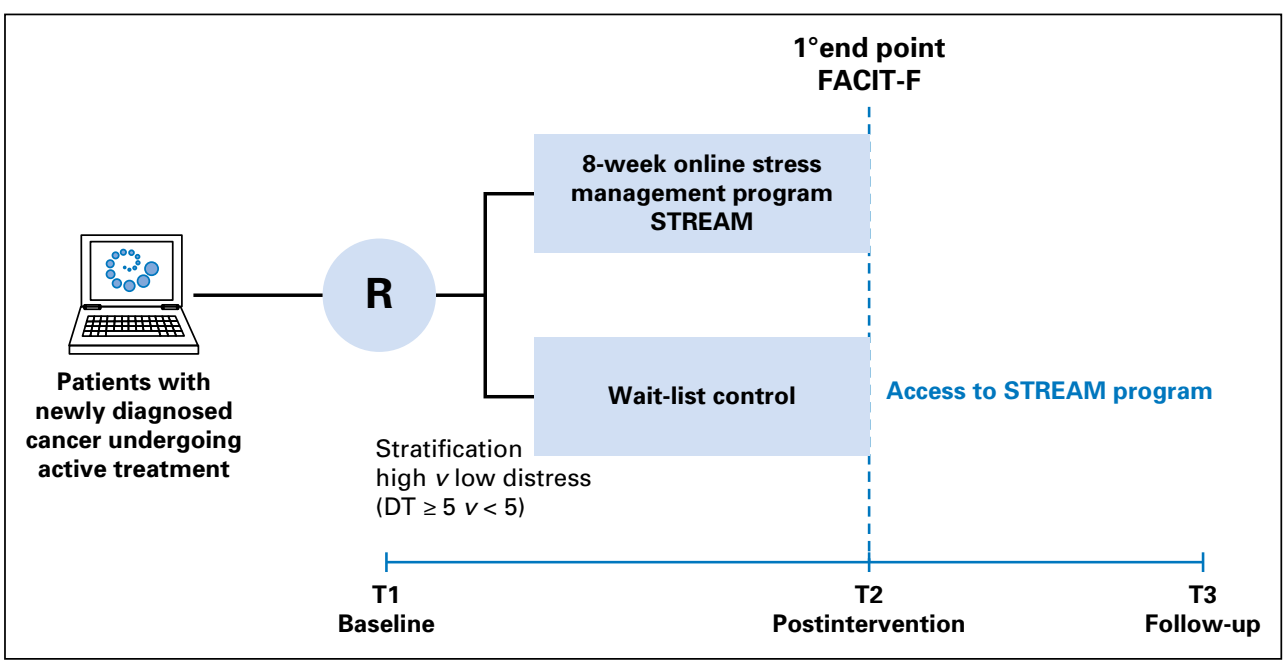

Fig 1. Trial design. DT, Distress Thermometer; FACIT-F, Functional Assessment of Chronic Illness Therapy-Fatigue; STREAM, Stress-Aktiv-Mindern. 
Secondary efficacy end points were assessed at the same points in time and evaluated psychological distress and anxiety or depression using the validated German versions of the National Comprehensive Cancer Network $\mathrm{DT}^{21}$ and the Hospital Anxiety and Depression Scale (HADS), ${ }^{25}$ respectively. Effect sizes are expressed as partial eta squared $\left(\eta_{p}^{2}\right),{ }^{26}$ with the following cutoffs to categorize effect sizes into small (0.01), medium $(0.06)$, and large $(0.14)$, as suggested by Cohen. ${ }^{27}$

\section{Assessments During Intervention}

Usability was evaluated after the first and last module with the System Usability Scale; scores $>70$ represent good usability. ${ }^{28}$ Therapeutic alliance between patients and the online therapist was assessed using the Working Alliance Inventory in its short form (12 items $)^{29}$ after each module. Total score ranges from 0 to 5 , and scores $>3.5$ have been rated as good working alliances. ${ }^{30}$

\section{Statistical Analyses and Sample Size Calculation}

All analyses were performed in the intention-to-treat (ITT) population defined as all patients who were randomly assigned. The perprotocol (PP) population included all patients who underwent the program in the intended timeframe (ie, the time between random assignment and $\mathrm{T} 2$ assessments did not exceed 16 weeks, which is twice the minimal duration of the program). To demonstrate a 9-point difference ${ }^{31}$ in FACIT-F total score between baseline and T2 (after 8 weeks) in the intervention group with a statistical power of 0.80 at a significance level of .05 (two sided), 60 participants were needed in each of the two conditions.

\section{Efficacy Analyses}

Efficacy outcomes were modeled with analysis of covariance (ANCOVA), using postscore (T2) as the dependent variable, prescore (T1) as the covariate, and group allocation (intervention $v$ control) as the independent variable. ANCOVAs were further adjusted for the stratification factor distress (DT $\geq 5 v<5$ ). For the follow-up period, score changes from T2 to T3 were analyzed with paired $t$ tests, separately for each group (no between-group comparisons). Multiple imputations $(\mathrm{n}=99)$ by chained equations ${ }^{32}$ using predictive mean matching ${ }^{33}$ incorporating all variables of the linear models underlying ANCOVA were used to impute missing outcome values. ${ }^{34}$ To assess the robustness of the results, sensitivity analyses were conducted for all outcomes in the PP population. In addition, sensitivity analyses were carried out using other methods for handling missing data; more specifically, complete-case analyses and last observation carried forward analyses, as specified in the protocol, ${ }^{20}$ were computed for all outcomes in both the ITT and PP populations.

\section{RESULTS}

We screened 229 patients, of whom 129 were randomly assigned between September 11, 2014, and November 24, 2016 (Fig 2). All patients received first-line cancer treatment, which they started a median of 17 days (interquartile range [IQR], 6-22 days) and 14 days (IQR, 7-20 days) after signing informed consent in the intervention and control groups, respectively. Patients were residents of Switzerland $(n=64)$, Germany $(n=59)$, Austria $(n=5)$, and the United Kingdom $(\mathrm{n}=1)$. Medical, psychological, and socioeconomic baseline characteristics are listed in Table 1 and were balanced between the groups. All 21 patients (control group, $\mathrm{n}=10$; intervention group, $\mathrm{n}=11$ ) who scored 1 point in the Beck Depression Inventory suicide item at baseline were immediately contacted by telephone, but they clearly distanced themselves from acute suicidal intent.

The intervention was designed to be feasible within 8 weeks. However, median duration of the online intervention (between first login to module one and postintervention assessment at T2) was 11.7 weeks (IQR, 9.1-18.6 weeks). In the intervention group, 52 patients $(80.0 \%)$ used at least six modules, and $49(75.4 \%)$ worked with all eight modules. Our psychologists spent a median time of 165 minutes (IQR, 127-210 minutes) for administering the online intervention (ie, 13.3 minutes [IQR, 9.5-17.9 minutes] per patient each week). Usability of the program was rated high, with a mean System Usability Scale score of 87.5 (IQR, 81.2-95.0) after module one and of 90.0 (IQR, 82.5-95.0) after module eight. As a measure of the therapeutic relationship between patient and online therapist, patients reported a mean score in the Working Alliance Inventory questionnaire of 3.77 (IQR, 3.38-4.14), similar to that of previously reported online working alliances. ${ }^{30}$

Primary and secondary efficacy outcomes are listed in Table 2 and illustrated in Figures 3 and 4. Quality of life (FACIT-F) after the intervention (T2; the primary end point) was significantly higher in the intervention group as compared with the control group (ANCOVA $P=.007$; Table 2 ). With a mean increase in total FACIT-F score of 8.59 (95\% CI, 2.45 to $14.73 ; P=.007)$ in the ITT population and of 10.71 (95\% CI, 4.49 to $16.94 ; P=.001)$ in the PP population, changes were clinically meaningful. ${ }^{19,24}$ Effect sizes were medium ${ }^{27}\left(\eta_{p}^{2}=0.063\right.$ and 0.114 in the ITT and PP populations, respectively; Table 2$)$. Increased scores within the fatigue (4.51; 95\% CI, 1.81 to $7.22 ; P=.002)$, physical well-being (2.01; $95 \% \mathrm{CI}, 0.43$ to $3.59 ; P=.01$ ), and functional well-being subscales $(1.53 ; 95 \% \mathrm{CI}, 0.11$ to $2.95 ; P=.04)$ were major contributors to the increase in total FACIT-F score, whereas social well-being and emotional well-being scores were not (Table 2.).

Distress on the VAS (scored from 0 to 10) of the National Comprehensive Cancer Network DT was significantly lower at T2 in the intervention group as compared with the control $(-0.85$; $95 \% \mathrm{CI},-1.60$ to $-0.10 ; P=.03)$. As summarized in Table 2, anxiety and depression (HADS) after the intervention (T2) were not significantly lower in the intervention as compared with the control group $(P=.15)$ in the ITT population. However, decrease in HADS score was statistically significant in the PP population $(-2.09 ; 95 \% \mathrm{CI},-4.03$ to $-0.16 ; P=.03)$. All results were confirmed in the prespecified sensitivity analyses (Appendix Table A2). Figure 4 shows the percentage of patients who reported any changes in scores between baseline and T2 for all three assessment tools.

During the 2-month follow-up period of the intervention group (T2 to T3), quality of life (FACIT-F T2 to T3: mean, 4.69; 95\% CI, -0.74 to $10.12 ; P=.09$ ), distress (DT T2 to T3: mean, $-0.29 ; 95 \% \mathrm{CI},-1.03$ to $0.44 ; P=.4$ ), and $\operatorname{mood}$ (HADS T2 to T3: mean, $-0.82 ; 95 \% \mathrm{CI},-2.28$ to $0.65 ; P=.27)$ did not change significantly. In the follow-up phase (ie, after T2), 51 $(79.7 \%)$ of 64 patients randomly assigned to the control arm opted to start the STREAM program. For this group of patients, T2 represents the assessments immediately before and T3 the assessments immediately after the online program. In an ITT analysis $(n=64)$, quality of life increased significantly (FACIT-F T2 to T3: mean, 10.95 ; $95 \% \mathrm{CI}, 6.18$ to $15.71 ; P<.001)$ and distress decreased significantly (DT T2 to T3: mean, -1.25 ; 95\% CI, -1.95 to $-0.55 ; P=.001$ ) between T2 and T3. Self-reported anxiety and depression were also lower (HADS T2 to T3: mean, $-2.83 ; 95 \%$ $\mathrm{CI},-4.29$ to $-1.36 ; P<.001)$. Again, results were confirmed in the prespecified sensitivity analyses (Appendix Table A3, online only). 
Data for individual patients and group means are shown in Appendix Figure A1 (online only).

\section{DISCUSSION}

In this randomized controlled trial, newly diagnosed patients with cancer reported significantly better quality of life and lower distress on the DT after participating in the therapist-guided Web-based stress management program STREAM. Recruitment to the STREAM study via online channels was successful, and patients in three countries, corresponding to a geographic area twice as large as the United Kingdom, were reached. Thus, dissemination of psychosocial interventions beyond urban centers, where face-to-face psychosocial interventions are available, ${ }^{35}$ can be facilitated by a Web-based approach. The STREAM intervention was feasible in our population of patients during a period of active treatment of different types of cancer with good adherence; $80 \%$ of patients worked with at least six of the eight modules. ${ }^{16}$

Although it is indisputable that quality of life matters, it is also inherently difficult to measure. ${ }^{36}$ To ensure robust and clinically relevant data, we rely on well-validated and standardized questionnaires. ${ }^{1,23,25}$

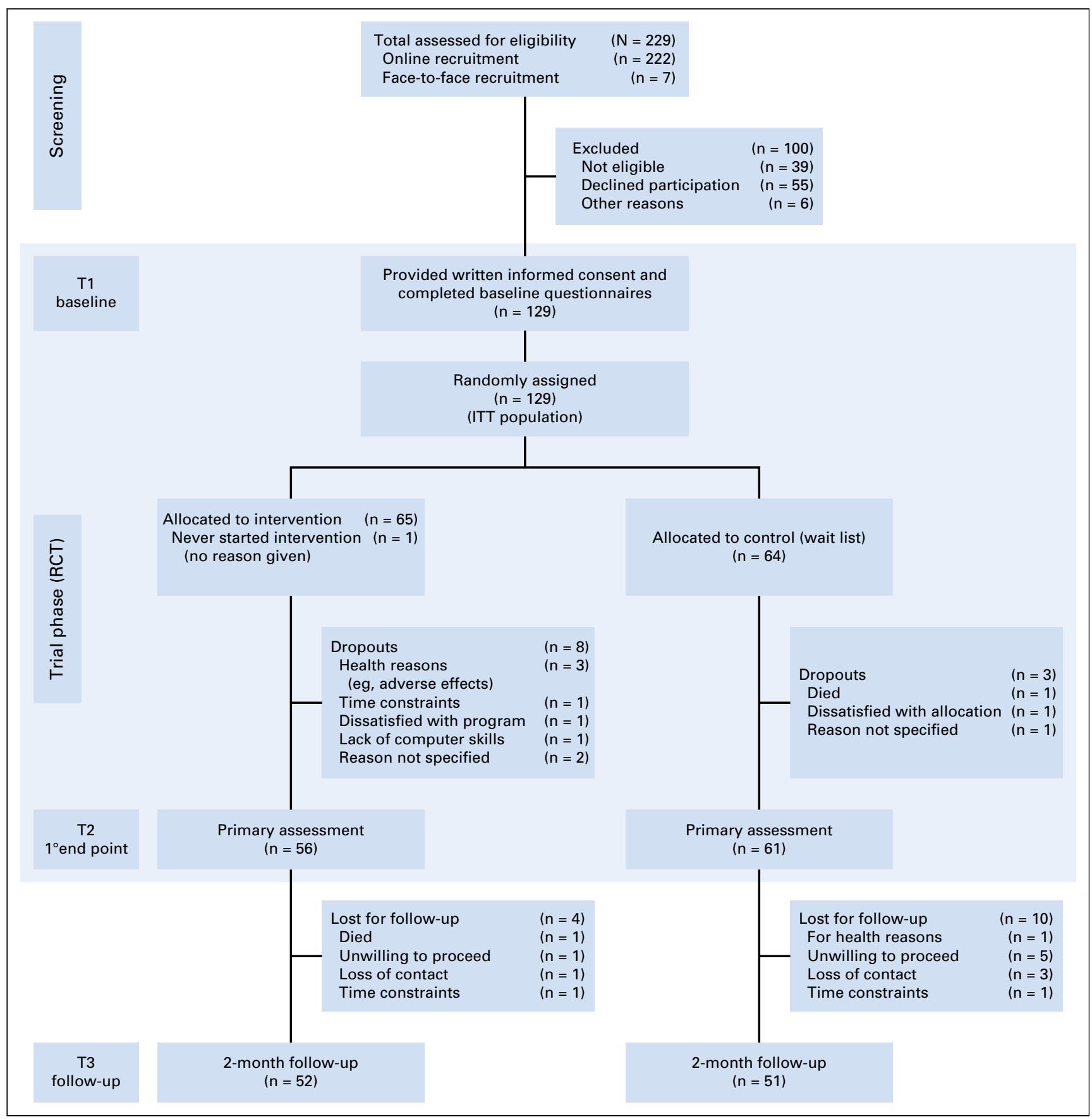

Fig 2. Patient flow (CONSORT diagram). ITT, intention to treat; $R C T$, randomized controlled trial. 


\begin{tabular}{|c|c|c|c|}
\hline \multirow[b]{2}{*}{ Characteristic } & \multicolumn{3}{|c|}{ No. $(\%)$} \\
\hline & $\begin{array}{l}\text { All Patients } \\
(\mathrm{N}=129)\end{array}$ & $\begin{array}{l}\text { Control } \\
\text { Group } \\
(n=64)\end{array}$ & $\begin{array}{l}\text { Intervention } \\
\text { Group } \\
(n=65)\end{array}$ \\
\hline \multicolumn{4}{|l|}{ Age, years } \\
\hline Median & 52 & 53 & 51 \\
\hline IQR & $46-58$ & $46-58$ & $46-57$ \\
\hline \multicolumn{4}{|l|}{ Sex } \\
\hline Female & 109 (84.5) & 56 (87.5) & 53 (81.5) \\
\hline Male & $20(15.5)$ & 8 (12.5) & $12(18.5)$ \\
\hline \multicolumn{4}{|l|}{ Tumor origin } \\
\hline Breast & $92(71.3)$ & 47 (73.4) & $45(69.2)$ \\
\hline Gynecologic tract & $7(5.4)$ & $5(7.8)$ & $2(3.1)$ \\
\hline Lung & $5(3.9)$ & $3(4.7)$ & $2(3.1)$ \\
\hline CNS/head and neck & $4(3.1)$ & $1(1.6)$ & $3(4.6)$ \\
\hline Lymphoma & $11(8.5)$ & $4(6.2)$ & $7(10.8)$ \\
\hline Skin/soft tissue & $1(0.8)$ & $1(1.6)$ & $0(0.0)$ \\
\hline $\mathrm{Gl}$ tract & $7(5.4)$ & $2(3.1)$ & $5(7.7)$ \\
\hline Urogenital tract & $2(2.0)$ & $1(1.6)$ & $1(1.5)$ \\
\hline \multicolumn{4}{|l|}{ Disease stage } \\
\hline Localized & $111(86.0)$ & 55 (85.9) & $56(86.2)$ \\
\hline Metastatic & $18(14.0)$ & $9(14.1)$ & 9 (13.8) \\
\hline \multicolumn{4}{|l|}{ Treatment } \\
\hline Radiotherapy & 16 (12.4) & $8(12.5)$ & 8 (12.3) \\
\hline Chemotherapy & $75(58.1)$ & $40(62.5)$ & $35(53.8)$ \\
\hline Hormonal therapy & $32(24.8)$ & 14 (21.9) & $18(27.7)$ \\
\hline Radiochemotherapy & $4(3.1)$ & $1(1.6)$ & $3(4.6)$ \\
\hline Other & $2(1.6)$ & $1(1.6)$ & $1(1.5)$ \\
\hline \multicolumn{4}{|l|}{ Treatment goal } \\
\hline Curative & $117(90.7)$ & 58 (90.6) & 59 (90.8) \\
\hline Palliative & $12(9.3)$ & $6(9.4)$ & $6(9.2)$ \\
\hline \multicolumn{4}{|l|}{ Marital status } \\
\hline Married & $81(62.8)$ & 39 (60.9) & $42(64.4)$ \\
\hline Married, separated & $2(1.6)$ & $1(1.6)$ & $1(1.5)$ \\
\hline Single & $26(20.2)$ & 12 (18.8) & $14(21.5)$ \\
\hline Divorced & 16 (12.4) & $9(14.1)$ & 7 (10.8) \\
\hline Widowed & $4(3.1)$ & $3(4.7)$ & $1(1.5)$ \\
\hline \multicolumn{4}{|l|}{ Highest education } \\
\hline Compulsory school & $2(1.6)$ & $1(1.6)$ & $1(1.5)$ \\
\hline Apprenticeship & $32(24.8)$ & $16(25.0)$ & 16 (24.6) \\
\hline College & $44(34.1)$ & $19(29.7)$ & 25 (38.5) \\
\hline University & $48(37.2)$ & $25(39.1)$ & $23(35.4)$ \\
\hline Other & $3(2.3)$ & $3(4.7)$ & $0(0.0)$ \\
\hline \multicolumn{4}{|c|}{$\begin{array}{l}\text { Monthly household income, } \\
\qquad(n=107)\end{array}$} \\
\hline$<1,200$ & $2(1.9)$ & $0(0.0)$ & $2(3.8)$ \\
\hline $1,200-2,500$ & $7(6.5)$ & $6(11.1)$ & $1(1.9)$ \\
\hline $2,500-4,900$ & 35 (32.7) & 18 (33.3) & $17(32.1)$ \\
\hline $4,900-8,100$ & $36(33.6)$ & $18(33.3)$ & $18(34.0)$ \\
\hline $8,100-12,200$ & 19 (17.8) & 9 (16.7) & 10 (18.9) \\
\hline$>12,200$ & $8(7.5)$ & $3(5.6)$ & $5(9.4)$ \\
\hline \multicolumn{4}{|l|}{$\begin{array}{l}\text { Using complementary } \\
\text { medicine } \\
\qquad(n=116)\end{array}$} \\
\hline Yes & $31(26.7)$ & $17(30.4)$ & $14(23.3)$ \\
\hline No & $51(44.0)$ & $23(41.1)$ & $28(46.7)$ \\
\hline I don't know & $34(29.3)$ & $16(28.6)$ & $18(30.0)$ \\
\hline \multicolumn{4}{|c|}{ Currently seeing a therapist } \\
\hline Yes & 45 (34.9) & $27(42.2)$ & $18(27.7)$ \\
\hline No & $84(65.1)$ & $37(57.8)$ & 47 (72.3) \\
\hline \multicolumn{4}{|c|}{$\begin{array}{l}\text { Currently using psychotropic } \\
\text { drugs }\end{array}$} \\
\hline Yes & $17(13.2)$ & $11(17.2)$ & $6(9.2)$ \\
\hline No & $111(86.0)$ & 53 (82.8) & $58(89.2)$ \\
\hline I don't know & $1(0.8)$ & $0(0)$ & $1(1.5)$ \\
\hline \multicolumn{4}{|c|}{ (continued in next column) } \\
\hline
\end{tabular}

\begin{tabular}{|c|c|c|c|}
\hline \multirow[b]{2}{*}{ Characteristic } & \multicolumn{3}{|c|}{ No. (\%) } \\
\hline & $\begin{array}{l}\text { All Patients } \\
(\mathrm{N}=129)\end{array}$ & $\begin{array}{l}\text { Control } \\
\text { Group } \\
(n=64)\end{array}$ & $\begin{array}{l}\text { Intervention } \\
\text { Group } \\
(n=65)\end{array}$ \\
\hline \multicolumn{4}{|l|}{ Baseline FACIT-F score } \\
\hline Median & 106.0 & 108.3 & 101.0 \\
\hline IQR & $84.2-123.0$ & $87.8-124.0$ & $81.0-120.0$ \\
\hline \multicolumn{4}{|l|}{ Baseline distress (DT) } \\
\hline Low & $30(23.3)$ & 14 (21.9) & $16(24.6)$ \\
\hline High (score $\geq 5$ ) & 99 (76.7) & $50(78.1)$ & 49 (75.4) \\
\hline \multicolumn{4}{|l|}{ Baseline HADS score } \\
\hline Median & 12 & 12 & 13 \\
\hline IQR & $7-17$ & $7-16$ & $7-18$ \\
\hline \multicolumn{4}{|c|}{$\begin{array}{l}\text { NOTE. No significant differences }(P<.05) \text { between groups were identified fo } \\
\text { any category (as determined by Wilcoxon or Kruskal-Wallis test or Pearsor } \\
\chi^{2} \text { test). } \\
\text { Abbreviations: DT, Distress Thermometer; FACIT-F, Functional Assessment o } \\
\text { Chronic lllness Therapy-Fatigue; HADS, Hospital Anxiety and Depression Scale } \\
\text { IQR, interquartile range. }\end{array}$} \\
\hline
\end{tabular}

There is no clear cutoff for clinically meaningful increases in overall quality of life in the FACIT-F score. However, on the basis of previous studies, changes reported in the postintervention scores of this trial were in a range that is considered highly noticeable to patients. ${ }^{19,24}$ Qualityof-life analyses are often complicated by a large number of missing data. In our study, the low number of missing data $(90.7 \%$ of all randomly assigned patients completed the primary assessment at T2) and robustness of the sensitivity analyses (Appendix Tables A2 and A3), increase reliability of patient-reported outcomes.

Although the primary efficacy end point of better quality of life after the STREAM intervention was clearly met, the effect of the intervention on distress is less clear cut. The DT is an assessment tool that allows patients to summarize all subjective aspects of distress in a single number (VAS, 0 to 10 ). In its simplicity, the DT therefore has the advantage of covering various dimensions of distress, including physical, functional, social, socioeconomic, spiritual, and emotional distress. ${ }^{21}$ However, the weight that patients assign, whether consciously or not, to each dimension is not discernible from the DT score. In contrast, the HADS questionnaire covers exclusively the emotional dimension of distress, but it does so in greater depth. ${ }^{25}$ Whereas self-reported distress on the DT was lower after STREAM, with a small to medium effect size ${ }^{27}$ $\left(\eta_{p}^{2}=0.043\right.$ and 0.069 in the ITT and PP populations, respectively), emotional distress as assessed by HADS did not change. This leads to the hypothesis that STREAM primarily affects dimensions of distress other than anxiety or depression. Of note, in our population, HADS scores at baseline were rather low (mean, 12; IQR, 7-17), whereas baseline DT scores were high (mean, 6; IQR, 5-8). It is therefore conceivable that a potential impact of STREAM on the emotional dimension of distress (anxiety and depression) cannot be assessed conclusively in our population. A study tailored specifically toward patients with high baseline levels of anxiety or depression would be more appropriate to answer this specific question.

Although STREAM was designed for and open to all newly diagnosed patients with cancer, women with breast cancer undergoing curative treatment represented the vast majority of the study population. This leaves uncertainty regarding generalizability 


\begin{tabular}{|c|c|c|c|c|c|c|}
\hline \multirow[b]{2}{*}{ Measure } & \multicolumn{3}{|c|}{$\begin{array}{l}\text { ITT Population } \\
\quad(n=129)\end{array}$} & \multicolumn{3}{|c|}{$\begin{array}{l}\text { PP Population* } \\
(n=95)\end{array}$} \\
\hline & $\Delta$ Meanst $(95 \% \mathrm{Cl})$ & $P$ & $E S\left(\eta_{p}^{2}\right) \neq$ & $\Delta$ Meanst $(95 \% \mathrm{Cl})$ & $P$ & $\operatorname{ES}\left(\eta_{p}^{2}\right) \neq$ \\
\hline Quality of life including fatigue (FACIT-F; 40 items; score, 0-160) & $8.59 \S(2.45$ to 14.73$)$ & .007 & 0.063 & 10.71 (4.49 to 16.94$)$ & .001 & 0.114 \\
\hline Physical well-being (seven items; score, 0-28) & 2.01 (0.43 to 3.59$)$ & .01 & & $2.64(1.02$ to 4.25$)$ & .002 & \\
\hline Social well-being (seven items; score, 0-28) & $0.44(-0.95$ to 1.82$)$ & .53 & & $0.41(-1.13$ to 1.96$)$ & .60 & \\
\hline Emotional well-being (six items; score, 0-24) & $0.24(-0.77$ to 1.25$)$ & .64 & & $0.68(-0.38$ to 1.75$)$ & .21 & \\
\hline Functional well-being (seven items; score, 0-28) & $1.53(0.11$ to 2.95$)$ & .04 & & $1.65(0.04$ to 3.26$)$ & .05 & \\
\hline Fatigue score (13 items; score, 0-52) & $4.52(1.81$ to 7.22$)$ & .002 & & $5.26(2.37$ to 8.16$)$ & .001 & \\
\hline DT (score, $0-10$ ) & $-0.85(-1.60$ to -0.10$)$ & .03 & 0.043 & $-1.11(-1.95$ to -0.26$)$ & .01 & 0.069 \\
\hline HADS (14 items; score, 0-42) & $-1.28(-3.02$ to 0.45$)$ & .15 & 0.019 & $-2.10(-4.03$ to -0.16$)$ & .03 & 0.049 \\
\hline \multicolumn{7}{|c|}{$\begin{array}{l}\text { NOTE. Results of analysis of covariance for postintervention scores (T2), with baseline scores (T1) as covariates, adjusted for baseline distress (stratification factor) } \\
\text { Abbreviations: DT, Distress Thermometer; ES, effect size; FACIT-F, Functional Assessment of Chronic Illness Therapy-Fatigue; HADS, Hospital Anxiety and Depression } \\
\text { Scale; ITT, intention to treat; PP, per protocol. } \\
\text { *PP population was defined as all patients for whom time between random assignment and T2 assessments did not exceed } 16 \text { weeks. } \\
\text { +Treatment effects estimated by analysis of covariance are reported as difference }(\Delta) \text { in scores of group means for intervention } v \text { control group. } \\
\text { fESs are expressed as partial eta squared, with the cutoffs small (0.01), medium }(0.06) \text {, and large }(0.14) \text {. Multiple imputations were used to handle missing data. Results } \\
\text { did not change significantly with other methods for handling of missing data (complete-case (CC) analysis or last observation carried forward (LOCF) analysis; Appendix } \\
\text { Table A2, online only). } \\
\text { \$Primary end point of the trial. }\end{array}$} \\
\hline
\end{tabular}

of the results, particularly toward men and toward the palliative setting. Women with breast cancer are known to have the largest social media network in the cancer community, which likely allowed for effective online recruitment. The presence of other cancer groups in the Internet community is only emerging, with platforms such as that created by the Movember Foundation for men with cancer. ${ }^{37}$ Such platforms may allow for integrating more men into future studies. If targeted specifically, men with prostate cancer also seem to be reachable via the Internet, as shown by an Australian self-help online program, which integrated a patient forum called My Road Ahead. ${ }^{38}$

At baseline, before random assignment, more patients in the control group reported face-to-face psychological support and use of psychotropic drugs than in the intervention group, although the number was not statistically significant. Data on the amount of time spent face to face with local psychologists during the course of the trial were not collected; hence, potential bias cannot be quantified. In contrast, attention bias toward the intervention group, possibly introduced by the time our STREAM psychologists spent online with the patients, may have affected outcome inversely. Because we opted for a care-as-usual (ie, wait-list) rather than active control, this will need to be differentiated in future studies.

A wait-list controlled design is generally accepted to control for the effect of time on the outcome of interest. However, the duration of the wait and consequently the timing of assessments (T2) for the control group are prospectively defined and rigid, whereas the timing of assessments (T2) in the interventions group is dependent on the duration of the intervention and therefore more variable. Hence, time sensitivity is only partially accounted for. This is also true for our study, where median time between T1 and T2 was 9.4 weeks (IQR, 8.6-12.1 weeks) for the intervention group but was shorter in the control group (median, 8.7 weeks; IQR, 8.3-9.3 weeks). Dynamic wait-list controlled designs have been proposed to minimize this potential bias. ${ }^{39}$

Another shortcoming of our trial is that we only show a benefit in distress and quality of life for patients early after

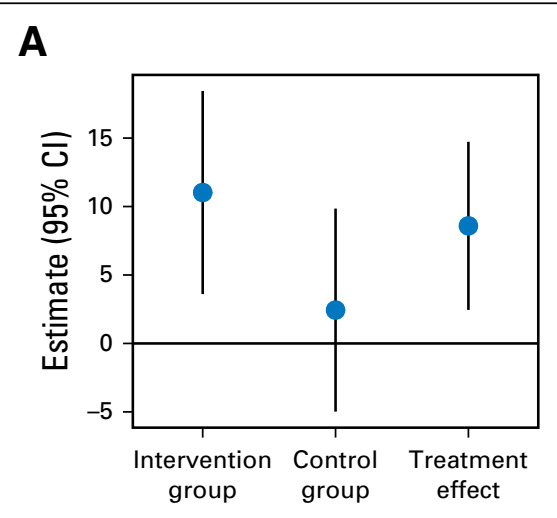

B

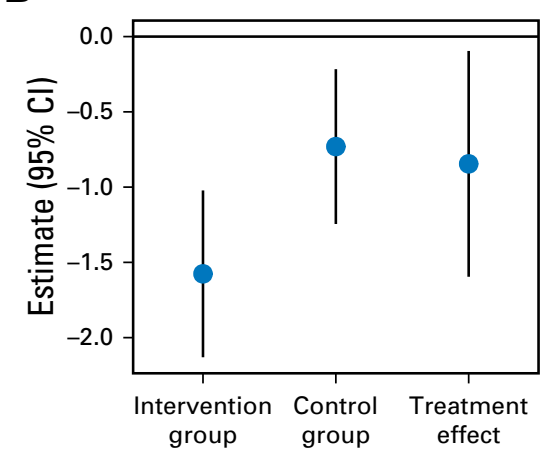

C

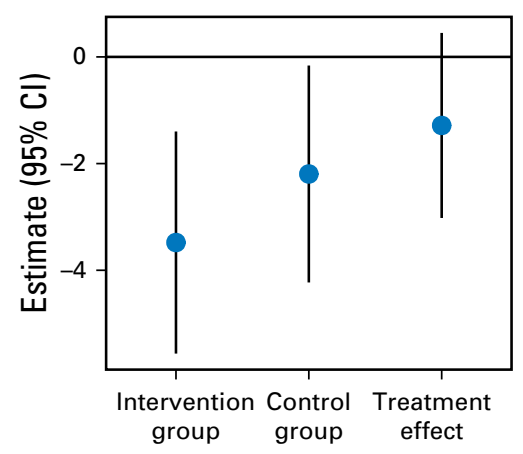

Fig 3. Treatment effects. Mean changes in scores (95\% Cls) postintervention (T2) for (A) quality of life (Functional Assessment of Chronic Illness Therapy-Fatigue), (B)

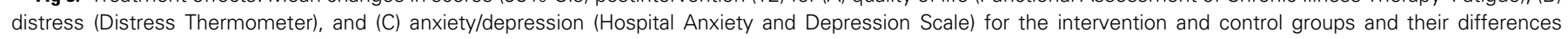
(treatment effects) based on analyses of covariance with prescores (T1) as covariates. 


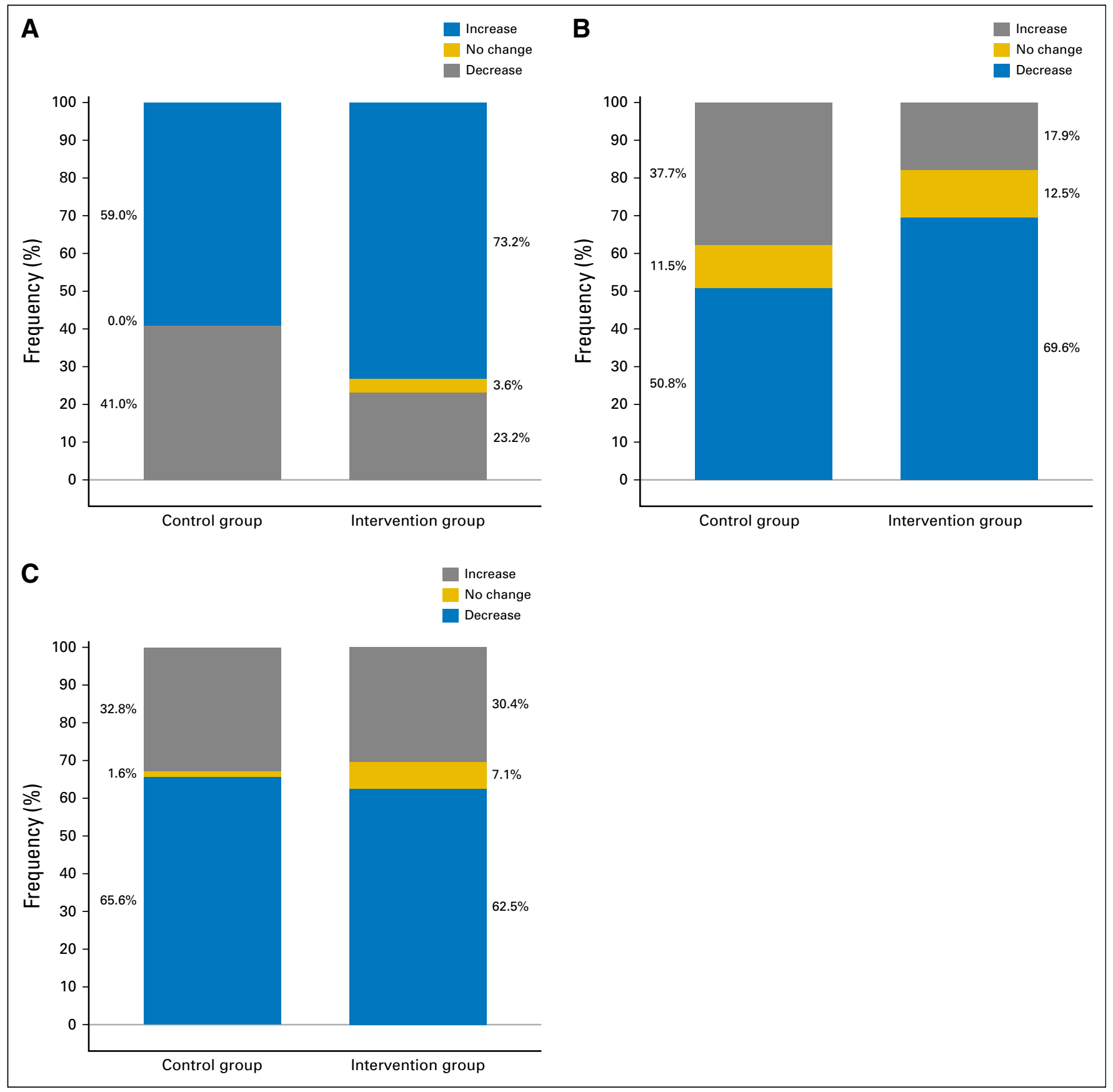

Fig 4. Percentage of patients with any increase or decrease or no change in total scores between $T 1$ and $T 2$ in (A) quality of life (Functional Assessment of Chronic Illness Therapy-Fatigue), (B) distress (Distress Thermometer), and (C) anxiety/depression (Hospital Anxiety and Depression Scale), where blue represents amelioration and gray deterioration within the respective assessment tool.

diagnosis, with a limited follow-up. It is conceivable, however, that such an early intervention ${ }^{19}$ may be of particular importance to prevent chronification of distress. ${ }^{40}$ Whether lower distress and increased quality of life after STREAM translate into better treatment tolerance and favorable disease course warrants additional studies.

The unique and common feature of study participants in this trial was a recent diagnosis of cancer. In contrast, the few reported randomized controlled trials on online support for patients with cancer have mainly focused on cancer survivors (ie, interventions later in the disease trajectory). In a randomized, wait-list controlled trial, breast cancer survivors (on average, 3 years after initial diagnosis) who participated in an online program in a similar therapist-guided format as presented here reported significantly improved sexual functioning (the primary end point of the trial) as compared with the wait-list control group. ${ }^{16}$ Breast cancer survivors were also the target population in the randomized trial for the Coping With Cancer Workbook. ${ }^{15}$ Women who participated in this Webbased self-help program reported better self-efficacy in coping with cancer. Overall quality of life was not reported. The BREATH (Breast 
Cancer eHealth) intervention, ${ }^{17}$ a Web-based intervention based on cognitive behavioral techniques but without therapist guidance, led to reduced distress in breast cancer survivors; however, it was not sustained during the 10-month follow-up.

In conclusion, with digital natives approaching an age that places them at risk for developing age-associated diseases, including cancer, use of the Internet in the health care setting will likely further increase. In this randomized trial, we found that a Web-based, guided self-help intervention resulted in a clinically meaningful improvement in quality of life. Our results indicate that Web-based, guided self-help has potential to efficiently support newly diagnosed patients with cancer.

\section{AUTHORS' DISCLOSURES OF POTENTIAL CONFLICTS OF INTEREST}

Disclosures provided by the authors are available with this article at jco.org.

\section{AUTHOR CONTRIBUTIONS}

Conception and design: Corinne Urech, Astrid Grossert, Judith Alder, Jens Gaab, Thomas Berger, Viviane Hess

Financial support: Corinne Urech, Viviane Hess

Administrative support: Corinne Urech, Astrid Grossert, Barbara Handschin, Viviane Hess

Provision of study materials or patients: Corinne Urech, Astrid Grossert, Sandra Scherer, Barbara Handschin, Borislava Borislavova, Sven Degen, Jennifer Erb, Alexandra Faessler, Sarah Schibli, Celine Werndli, Viviane Hess Collection and assembly of data: Corinne Urech, Astrid Grossert, Judith Alder, Sandra Scherer, Barbara Handschin, Benjamin Kasenda, Borislava Borislavova, Sven Degen, Jennifer Erb, Alexandra Faessler, Laura Gattlen, Sarah Schibli, Celine Werndli, Thomas Berger, Thomas Zumbrunn,

Viviane Hess

Data analysis and interpretation: Corinne Urech, Astrid Grossert, Judith Alder, Benjamin Kasenda, Thomas Berger, Thomas Zumbrunn, Viviane Hess

Manuscript writing: All authors

Final approval of manuscript: All authors

Accountable for all aspects of the work: All authors

\section{REFERENCES}

1. Mehnert A, Brähler $E$, Faller $H$, et al: Fourweek prevalence of mental disorders in patients with cancer across major tumor entities. J Clin Oncol 32: 3540-3546, 2014

2. Duijts SF, Faber MM, Oldenburg HS, et al: Effectiveness of behavioral techniques and physical exercise on psychosocial functioning and healthrelated quality of life in breast cancer patients and survivors: A meta-analysis. Psychooncology 20: 115-126, 2011

3. Luebbert K, Dahme B, Hasenbring M: The effectiveness of relaxation training in reducing treatment-related symptoms and improving emotional adjustment in acute non-surgical cancer treatment: $A$ meta-analytical review. Psychooncology 10:490-502, 2001

4. Kim GM, Kim SJ, Song SK, et al: Prevalence and prognostic implications of psychological distress in patients with gastric cancer. BMC Cancer 17:283, 2017

5. Stagl JM, Lechner SC, Carver CS, et al: A randomized controlled trial of cognitive-behavioral stress management in breast cancer: Survival and recurrence at 11-year follow-up. Breast Cancer Res Treat 154:319-328, 2015

6. Carlson LE, Angen M, Cullum J, et al: High levels of untreated distress and fatigue in cancer patients. Br J Cancer 90:2297-2304, 2004

7. Waller $A$, Williams $A$, Groff $S L$, et al: Screening for distress, the sixth vital sign: Examining selfreferral in people with cancer over a one-year period. Psychooncology 22:388-395, 2013

8. van de Poll-Franse LV, van Eenbergen MC: Internet use by cancer survivors: Current use and future wishes. Support Care Cancer 16:1189-1195, 2008

9. Andersson G: Internet-delivered psychological treatments. Annu Rev Clin Psychol 12:157-179, 2016

10. Kato PM, Cole SW, Bradlyn AS, et al: A video game improves behavioral outcomes in adolescents and young adults with cancer: A randomized trial. Pediatrics 122:e305-e317, 2008
11. Kessler D, Lewis G, Kaur S, et al: Therapistdelivered Internet psychotherapy for depression in primary care: A randomised controlled trial. Lancet 374:628-634, 2009

12. Buntrock C, Ebert DD, Lehr D, et al: Effect of a Web-based guided self-help intervention for prevention of major depression in adults with subthreshold depression: A randomized clinical trial. JAMA 315:1854-1863, 2016

13. Andersson G, Cuijpers $P$, Carlbring $P$, et al: Guided Internet-based vs. face-to-face cognitive behavior therapy for psychiatric and somatic disorders: A systematic review and meta-analysis. World Psychiatry 13:288-295, 2014

14. Beatty L, Koczwara B, Wade T: Evaluating the efficacy of a self-guided Web-based CBT intervention for reducing cancer-distress: A randomised controlled trial. Support Care Cancer 24:1043-1051, 2016

15. Carpenter KM, Stoner SA, Schmitz K, et al: An online stress management workbook for breast cancer. J Behav Med 37:458-468, 2014

16. Hummel SB, van Lankveld JJDM, Oldenburg HSA, et al: Efficacy of Internet-based cognitive behavioral therapy in improving sexual functioning of breast cancer survivors: Results of a randomized controlled trial. J Clin Oncol 35:1328-1340, 2017

17. van den Berg SW, Gielissen MF, Custers JA, et al: BREATH: Web-based self-management for psychological adjustment after primary breast cancer-Results of a multicenter randomized controlled trial. J Clin Oncol 33:2763-2771, 2015

18. Fang F, Fall K, Mittleman MA, et al: Suicide and cardiovascular death after a cancer diagnosis. N Engl J Med 366:1310-1318, 2012

19. Temel JS, Greer JA, Muzikansky A, et al: Early palliative care for patients with metastatic non-smallcell lung cancer. N Engl J Med 363:733-742, 2010

20. Grossert A, Urech C, Alder J, et al: Web-based stress management for newly diagnosed cancer patients (STREAM-1): A randomized, wait-list controlled intervention study. BMC Cancer 16:838, 2016

21. Mehnert A, Muller D, Lehmann $C$, et al: The German version of the NCCN Distress Thermometer: Validation of a screening instrument for assessment of psychosocial distress in cancer patients. Z Psychiatr Psychol Psychother 54:213-223, 2006
22. Antoni $M H$, Lechner $S$, Diaz $A$, et al: Cognitive behavioral stress management effects on psychosocial and physiological adaptation in women undergoing treatment for breast cancer. Brain Behav Immun 23:580-591, 2009

23. Webster K, Cella D, Yost K: The Functional Assessment of Chronic Illness Therapy (FACIT) measurement system: Properties, applications, and interpretation. Health Qual Life Outcomes 1:79, 2003

24. Cella D, Eton DT, Lai JS, et al: Combining anchor and distribution-based methods to derive minimal clinically important differences on the Functional Assessment of Cancer Therapy (FACT) anemia and fatigue scales. J Pain Symptom Manage 24:547-561, 2002

25. Zigmond AS, Snaith RP: The Hospital Anxiety and Depression Scale. Acta Psychiatr Scand 67: 361-370, 1983

26. Lakens D: Calculating and reporting effect sizes to facilitate cumulative science: A practical primer for t-tests and ANOVAs. Front Psychol 4:863, 2013

27. Cohen J: Statistical Power Analysis for the Behavioral Sciences (ed 2). Hillsdale, NJ, Lawrence Erlbaum Associates, 1988

28. Kortum $P$, Bangor $A$ : Usability ratings for everyday products measured with the System Usability Scale. Int J Hum Comput Interact 29:67-76, 2013

29. Hatcher RL, Gillaspy AJ: Development and validation of a revised short version of the Working Alliance Inventory. Psychother Res 16:12-25, 2006

30. Berger T: The therapeutic alliance in internet interventions: A narrative review and suggestions for future research. Psychother Res 27:511-524, 2017

31. Naumann F, Munro A, Martin E, et al: An individual-based versus group-based exercise and counselling intervention for improving quality of life in breast cancer survivors: A feasibility and efficacy study. Psychooncology 21:1136-1139, 2012

32. Raghunathan TE, Lepkowski JM, Van Hoewyk $\mathrm{J}$, et al: A multivariate technique for multiply imputing missing values using a sequence of regression models. Surv Methodol 27:85-95, 2001

33. van Buuren S: Multiple imputation of discrete and continuous data by fully conditional specification. Stat Methods Med Res 16:219-242, 2007 
34. Little R: Missing-data adjustments in large surveys. J Bus Econ Stat 6:287-296, 1988

35. Kessler RC, Demler O, Frank RG, et al: Prevalence and treatment of mental disorders, 1990 to 2003. N Engl J Med 352:2515-2523, 2005

36. Testa MA, Simonson DC: Assessment of qualityof-life outcomes. N Engl J Med 334:835-840, 1996
37. Movember Foundation. https://uwww.movember. com/

38. Wootten AC, Abbott JA, Meyer D, et al: Preliminary results of a randomised controlled trial of an online psychological intervention to reduce distress in men treated for localised prostate cancer. Eur Urol 68:471-479, 2015
39. Brown $\mathrm{CH}$, Wyman PA, Guo J, et al: Dynamic wait-listed designs for randomized trials: New designs for prevention of youth suicide. Clin Trials 3:259-271, 2006

40. Enns A, Waller A, Groff SL, et al: Risk factors for continuous distress over a 12-month period in newly diagnosed cancer outpatients. J Psychosoc Oncol 31:489-506, 2013

\section{Affiliations}

Corinne Urech, Astrid Grossert, Sandra Scherer, Barbara Handschin, Benjamin Kasenda, and Viviane Hess, University Hospital Basel; Astrid Grossert, Judith Alder, Barbara Handschin, Benjamin Kasenda, Borislava Borislavova, Sven Degen, Jennifer Erb, Alexandra Faessler, Laura Gattlen, Sarah Schibli, Celine Werndli, Jens Gaab, Thomas Zumbrunn, and Viviane Hess, University of Basel, Basel; and Thomas Berger, University of Bern, Bern, Switzerland.

\section{Support}

Supported by the Swiss National Science Foundation (Grant No. SNSF PP00P3_139155/1) and Cancer Research Switzerland (Grant No. KFS-3260-08-2013).

\section{Prior Presentation}

Presented in part as a late-breaking abstract at the 53rd Annual Meeting of the American Society of Clinical Oncology, Chicago, IL, June 2-6, 2017.

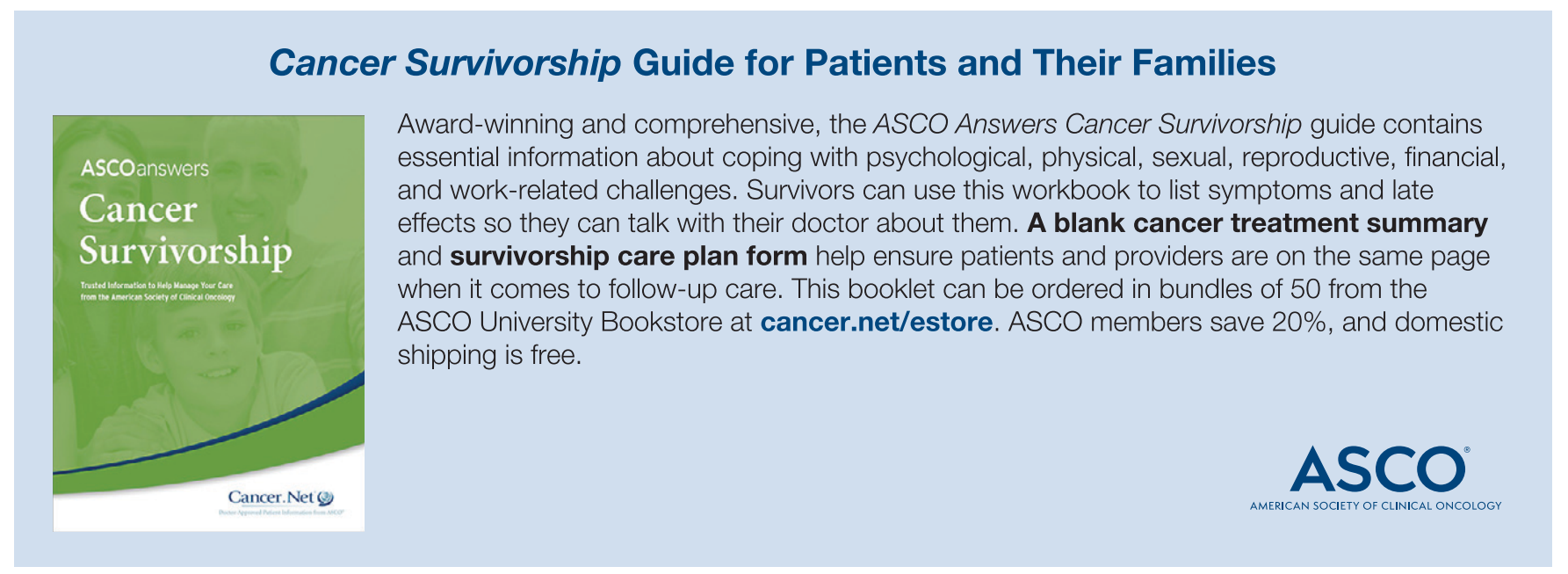




\section{AUTHORS' DISCLOSURES OF POTENTIAL CONFLICTS OF INTEREST}

Web-Based Stress Management for Newly Diagnosed Patients With Cancer (STREAM): A Randomized, Wait-List Controlled Intervention Study

The following represents disclosure information provided by authors of this manuscript. All relationships are considered compensated. Relationships are self-held unless noted. I = Immediate Family Member, Inst = My Institution. Relationships may not relate to the subject matter of this manuscript. For more information about ASCO's conflict of interest policy, please refer to www.asco.org/rwc or ascopubs.org/jco/site/ifc.

\section{Corinne Urech}

No relationship to disclose

\section{Astrid Grossert}

No relationship to disclose

\section{Judith Alder}

No relationship to disclose

\section{Sandra Scherer}

No relationship to disclose

\section{Barbara Handschin}

Employment: Novartis (I)

Stock or Other Ownership: Novartis (I)

Patents, Royalties, Other Intellectual Property: Novartis (I)

\section{Benjamin Kasenda}

No relationship to disclose

\section{Borislava Borislavova}

No relationship to disclose

\section{Sven Degen}

No relationship to disclose

\section{Jennifer Erb}

No relationship to disclose

Alexandra Faessler

No relationship to disclose

\section{Laura Gattlen}

No relationship to disclose

Sarah Schibli

No relationship to disclose

Celine Werndli

No relationship to disclose

Jens Gaab

No relationship to disclose

Thomas Berger

No relationship to disclose

Thomas Zumbrunn

No relationship to disclose

Viviane Hess

Travel, Accommodations, Expenses: Amgen 


\section{Acknowledgment}

We thank the software company YooApplication AG, Basel, Switzerland, for its collaboration in development and maintenance of the STREAM application. We thank Eva Oertle for professional recording of the audio files in STREAM and Lucia Uebersax for public relations work to foster recruitment.

\section{Appendix}

\section{Patients and Methods}

\section{Patient Eligibility Criteria and Recruitment}

Rationale and design of this randomized trial have been published. ${ }^{20}$ We included adult patients (age $\geq 18$ years) with newly diagnosed cancer who started first-line treatment (either systemic treatment, including chemotherapy, hormonal treatment, or targeted therapy, or radiotherapy) no longer than 12 weeks before study registration. Patients were required to provide written informed consent, read and write in German, have Internet access, and have basic computer skills. To obtain informed consent, a therapist provided detailed information about the study via telephone and went point by point through the informed consent form. The informed consent form was then sent to the patient, who, if in agreement, sent the signed paper form back via post mail. The Ethics Committee of Northwestern and Central Switzerland approved the study (EKNZ 339/13). The trial is registered at ClinicalTrials.gov (ClinicalTrials.gov identifier: NCT02289014).

For safety reasons, patients were assessed at baseline for suicidal tendency by the suicide item of the Beck Depression Inventory (Green KL, et al: J Clin Psychiatry 76:1683-1686, 2015). Our online program was not designed to support suicidal patients in acute crises; therefore, patients with a score higher than 1 were contacted by telephone and referred to immediate local support.

Information on medical history was obtained from the patients during baseline assessments and confirmed by their treating physicians, who we contacted by e-mail or telephone.

\section{Patient Recruitment}

Patients were recruited via the public Web site of STREAM (Stress-Aktiv-Mindern). To foster recruitment, we linked and distributed information about the trial via the following channels: links from health-related Web sites, such as cancer leagues, cancer hospitals, and patient advocate Web sites to the public Web site of STREAM; distribution of flyers in hospitals and during cancer conferences; communication to medical lay press; and paid advertisement via Google and Facebook.

\section{Study Design}

We randomly assigned eligible patients at a ratio of one to one using blocked randomization with randomly selected block sizes to an intervention group or a wait-list control group (Fig 1). Patients were stratified according to baseline distress, using an internationally accepted cutoff of $\geq 5$ points on the 10-point visual analog scale of the Distress Thermometer. ${ }^{21}$

\section{Intervention}

We developed the Web-based intervention STREAM ${ }^{20}$ based on established stress management intervention manuals ${ }^{22}$ that incorporate cognitive behavioral- and mindfulness-based stress reduction techniques, which we adapted to the Web context. STREAM aims at improving intra- and interpersonal coping strategies, thereby reducing perceived stress, anxiety, and fatigue as well as enhancing quality of life. STREAM consists of eight modules (Appendix Table A1), which can be completed in 60 to 90 minutes each. Each module starts with a short mindfulness breathing exercise followed by text-based psychoeducation, reflection on current individual emotional status, and acquisition of coping strategies including several exercises and worksheets. Daily use of downloadable audio files with relaxation and guided-imagery exercises was encouraged. Participants were asked to complete one module per week. Access to the next module was provided after the weekly online feedback of a therapist. Patients who had to interrupt the program for medical or personal reasons were regularly contacted and offered continuation. The total duration of the program was not limited. Therapists monitored participants' progress in the program and contacted the participants via an integrated and secured e-mail system to provide feedback and structure. Patients could use the integrated e-mail system to contact their therapist whenever they felt the need to and were informed that the therapist would answer within 3 working days. Whenever patients did not log in to the program for 7 days, therapists sent an e-mail reminder.

Therapists were four female psychologists with Master's degrees in clinical psychology and clinical experience in psychooncology. Three of them were in postgraduate psychotherapy training programs, and one was a licensed psychotherapist (C.U.). This core team was supported by seven psychologists with Bachelor's degrees in psychology, who worked under supervision of the 
licensed psychotherapist. All therapists were trained by the psychological study team leaders (A.G., C.U.) in applying the STREAM program and met for weekly discussions, with input by a medical oncologist (V.H.), to align procedures and strategies. For data protection, the program is password protected and secured by Secure Socket Layer encryption.

\section{Control}

Patients in the control group underwent their cancer treatment locally as planned and were recontacted by the study team 8 weeks after random assignment (T2; Fig 1). After T2 assessments, they received access to the online program. For patients in both groups, cancer treatment was determined locally, and supportive care, according to local standards, may also have included face-toface psychosocial support and psychotropic drugs.

\section{Assessments}

Assessments were conducted electronically directly within the Web-based program via the open source application LimeSurvey at baseline (T1) and after the intervention or waiting period (control group), respectively (T2). In addition, 2-month follow-up (T3) was performed in both groups. Feasibility was assessed at two different levels: feasibility of online recruitment and feasibility for patients to participate in the STREAM program while undergoing first-line treatment. According to the study protocol, feasibility of online recruitment was confirmed when 120 patients (ie, the number needed to assess the primary efficacy outcome) were recruited within a 2-year period; feasibility of participation during treatment was prespecified as a descriptive end point-more specifically, the percentage of completers' in the intention-to-treat (ITT) population, where completers' were defined as patients who worked with at least six of eight modules.

\section{Efficacy End Points}

Primary end point was quality of life at T2, assessed in the validated German version of the Functional Assessment of Chronic Illness Therapy-Fatigue (FACIT-F) questionnaire. ${ }^{23}$ The first 27 items are common to all Functional Assessment of Cancer Therapy (FACT) questionnaires and cover different domains of quality of life, specifically physical well-being (seven items), social well-being (seven items), emotional well-being (six items), and functional well-being (seven items). The last 13 items focus on various aspects of fatigue, a key aspect of quality of life in patients with cancer, particularly during active treatment. ${ }^{6}$ FACIT-F total score ranges from 0 to 160. Higher scores represent better quality of life. Minimal clinically meaningful differences are not well defined but have previously been set between 7 and 9 points, both as intraindividual changes and differences in groups. ${ }^{19,24}$ For our sample size calculation, we relied on the more stringent definition of 9 points. We chose FACIT-F, rather than the distress thermometer (DT), as primary outcome because overall well-being, reflected by the multidimensional FACIT-F questionnaire, seems clinically more important than changes in a single domain. ${ }^{23}$ Also, FACIT-F is better validated as outcome measure than DT, which often serves as screening tool. ${ }^{21}$

Secondary efficacy end points were assessed at the same points in time and evaluated psychological distress and anxiety and depression using the validated German versions of the National Comprehensive Cancer Network DT ${ }^{21}$ and the Hospital Anxiety and Depression Scale (HADS), ${ }^{25}$ respectively. Effect sizes are expressed as partial eta squared $\left(\eta_{\mathrm{p}}^{2}\right){ }^{26}$ with the following cutoffs to categorize effect sizes into small (0.01), medium (0.06), and large (0.14), as suggested by Cohen. ${ }^{27}$ Subgroup analyses are highly explorative and, therefore, not part of this report.

\section{Assessments During the Intervention}

Usability was evaluated after the first and last module with the System Usability Scale (SUS), where scores $>70$ represent good usability. ${ }^{28}$ Therapeutic alliance between patients and online therapist was assessed using the Working Alliance Inventory (WAI) in its short form (12 items), ${ }^{29}$ adapted with 2 additional items specific to the online context after each module. Total score ranges from 0 to 5 , and scores $>3.5$ have been rated as good working alliances. ${ }^{30}$

\section{Statistical Analyses and Sample Size Calculation}

$\mathrm{R}$ version 3.4.0 software (R Foundation for Statistical Computing, Vienna, Austria) was used for all analyses. All analyses were performed in the ITT population defined as all patients who were randomly assigned. The per-protocol (PP) population included all patients who completed the program in the intended timeframe (ie, the time between random assignment and T2 assessments did not exceed 16 weeks, which is twice the minimal duration of the program).

To demonstrate a 9-point difference ${ }^{31}$ in FACIT-F total score between baseline and T2 (after 8 weeks) in the intervention group with a statistical power of 0.80 at a significance level of .05 (two sided), 60 participants were needed in each of the two conditions. We assumed normally distributed data in both groups with a standard deviation of \pm 18 (Pandey M, et al: World J Surg Oncol 3:63, 
2005). Data preparation of all continuous dependent variables included tests for normality, homogeneity of variances, and examination of outliers.

\section{Efficacy Analyses}

Efficacy outcomes (primary end point of FACIT-F; secondary end points of DT and HADS) were modeled with analysis of covariance (ANCOVA), using the postscore (T2) as dependent variable, the prescore (T1) as covariate, and group allocation (intervention $v$ control) as independent variable. ANCOVAs were further adjusted for the stratification factor distress (DT $\geq 5 v<5$ ). Multiple imputations $(n=99)$ by chained equations ${ }^{32}$ using predictive mean matching ${ }^{33}$ incorporating all variables of the linear models underlying ANCOVA were used to impute missing outcome values. ${ }^{34}$ To assess the robustness of the results, sensitivity analyses were conducted for all outcomes in the perprotocol population. In addition, sensitivity analyses were carried out using other methods for handling missing data; more specifically, CC analyses and LOCF analyses, as specified in the protocol, ${ }^{20}$ were computed for all outcomes on both the ITT and PP populations.

\section{2-Month Follow-Up Analyses}

For the follow-up period, score changes from T2 to T3 were analyzed with paired $t$ tests separately for each group (no betweengroup comparisons). Again, multiple imputations were used for missing data. Sensitivity analyses were conducted using CC and LOCF analyses in the ITT and PP populations.

\section{Role of Funding Bodies}

The funder of the study had no role in study design, data collection, data analysis, data interpretation, or writing of the report. The corresponding author had full access to all the data in the study and had final responsibility for the decision to submit for publication.

\begin{tabular}{|c|c|c|c|}
\hline Module & Psychoeducation & Reflection on Status & Strategies and Exercises \\
\hline Introduction: What is stress? & $\begin{array}{l}\text { Nature of stress in general and specifically } \\
\text { in relation to cancer }\end{array}$ & My individual stressors & Diary, ${ }^{*}$ body scant \\
\hline Bodily stress reduction & $\begin{array}{l}\text { Bodily sensations during stress and } \\
\text { adverse effects of anticancer treatment, } \\
\text { focus on fatigue }\end{array}$ & $\begin{array}{l}\text { My individual bodily stress } \\
\text { reactions }\end{array}$ & Stress protocol* \\
\hline Cognitive stress reduction & $\begin{array}{l}\text { Thoughts and their interaction with } \\
\text { emotions and bodily sensations }\end{array}$ & My negative thought patterns & Progressive muscle relaxationt \\
\hline Emotional stress reduction & $\begin{array}{l}\text { Feelings and cancer-related emotions } \\
\text { such as anxiety and worries }\end{array}$ & My feelings and worries & $\begin{array}{l}\text { Walk on the beach, } \uparrow \text { relaxation protocol, } \\
\text { negative thought cycle, } \uparrow \text { relationship of } \\
\text { body position and thoughts, }{ }^{*} \text { thinking } \\
\text { styles and reflection* }\end{array}$ \\
\hline $\begin{array}{l}\text { Mindfulness and acceptance of thoughts } \\
\text { and emotions }\end{array}$ & $\begin{array}{l}\text { Meaning and implementation of } \\
\text { mindfulness and acceptance in daily life } \\
\text { (as opposed to active strategies learned } \\
\text { in modules one to four) }\end{array}$ & $\begin{array}{l}\text { My definition and experiences } \\
\text { with acceptance }\end{array}$ & $\begin{array}{l}\text { Thoughts on clouds, } \uparrow \text { mountain } \\
\text { meditation, } \dagger \text { emotional emergency kit } \dagger\end{array}$ \\
\hline $\begin{array}{l}\text { Activation of resources: quality of life and } \\
\text { pleasure }\end{array}$ & $\begin{array}{l}\text { Introduction of models for balance } \\
\text { between burden and resources }\end{array}$ & My individual resources & Acceptance story* $\dagger$ \\
\hline $\begin{array}{l}\text { Activation of resources: social network } \\
\text { and communication skills }\end{array}$ & $\begin{array}{l}\text { Social network and the role of a supportive } \\
\text { environment }\end{array}$ & $\begin{array}{l}\text { My individual social network and } \\
\text { current needs }\end{array}$ & Body scan* + \\
\hline Summary & $\begin{array}{l}\text { Overview and documentation of the last } \\
7 \text { weeks }\end{array}$ & $\begin{array}{l}\text { My experiences with the } \\
\text { program }\end{array}$ & $\begin{array}{l}\text { Integration of mindfulness, }{ }^{*} \text { winter walk, } \dagger \\
\text { spring awakening, } \dagger \text { health cycle, }{ }^{*} \\
\text { planning activities, }{ }^{*} \text { week planner, }{ }^{*} \\
\text { friendly feelings toward our own body, } \dagger \\
\text { enjoyment training, }{ }^{*} \text { communication } \\
\text { skills, }{ }^{*} \text { walk on the beach, } \dagger \text { winter } \\
\text { walk, } \dagger \text { spring awakening, } \dagger \text { four } \\
\text { seasons } \dagger\end{array}$ \\
\hline \multicolumn{4}{|c|}{$\begin{array}{l}\text { Abbreviation: STREAM, Stress-Aktiv-Mindern. } \\
\text { *Instructions and worksheets. } \\
\text { †Audio file: story, relaxation, or guided imaginary exercise. }\end{array}$} \\
\hline
\end{tabular}




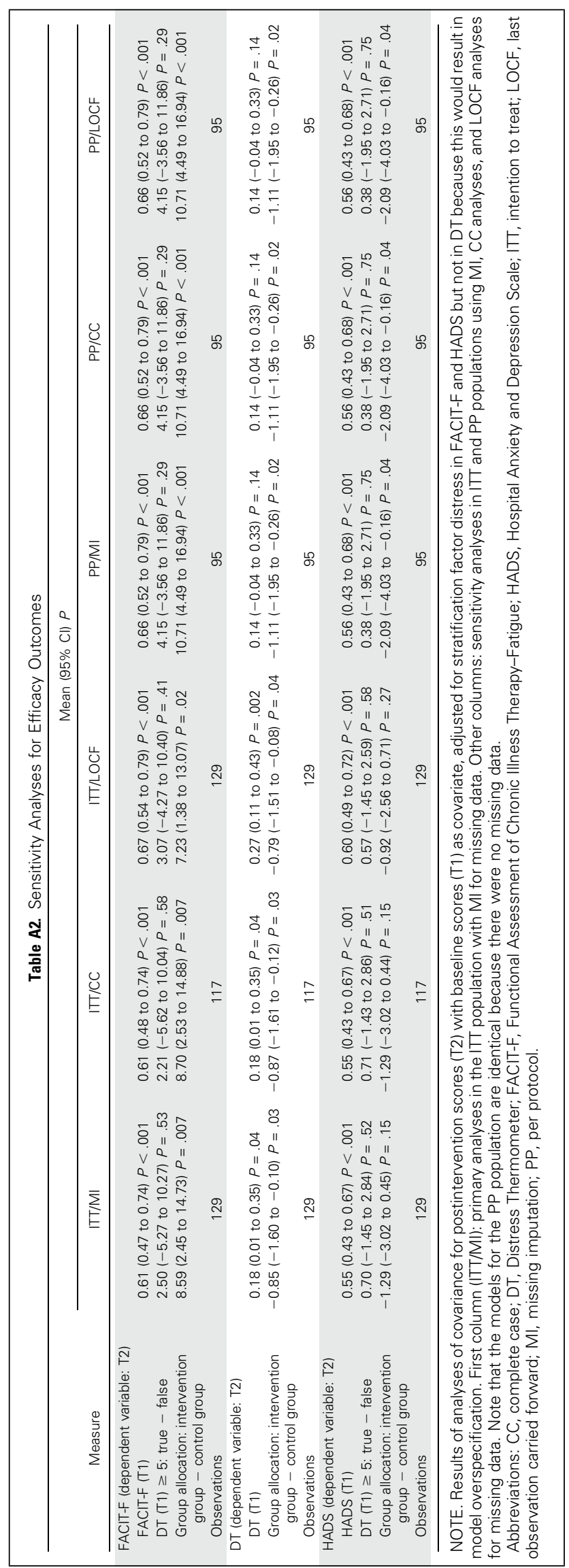




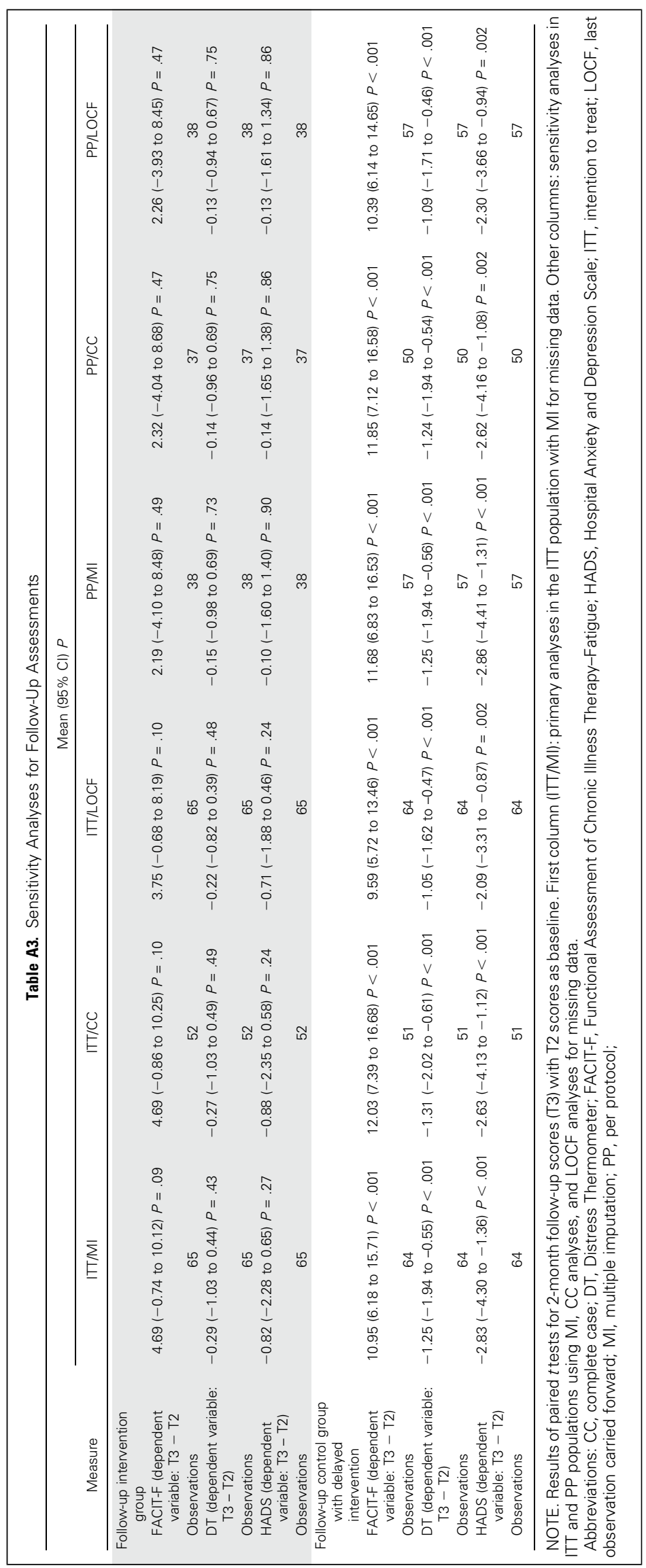




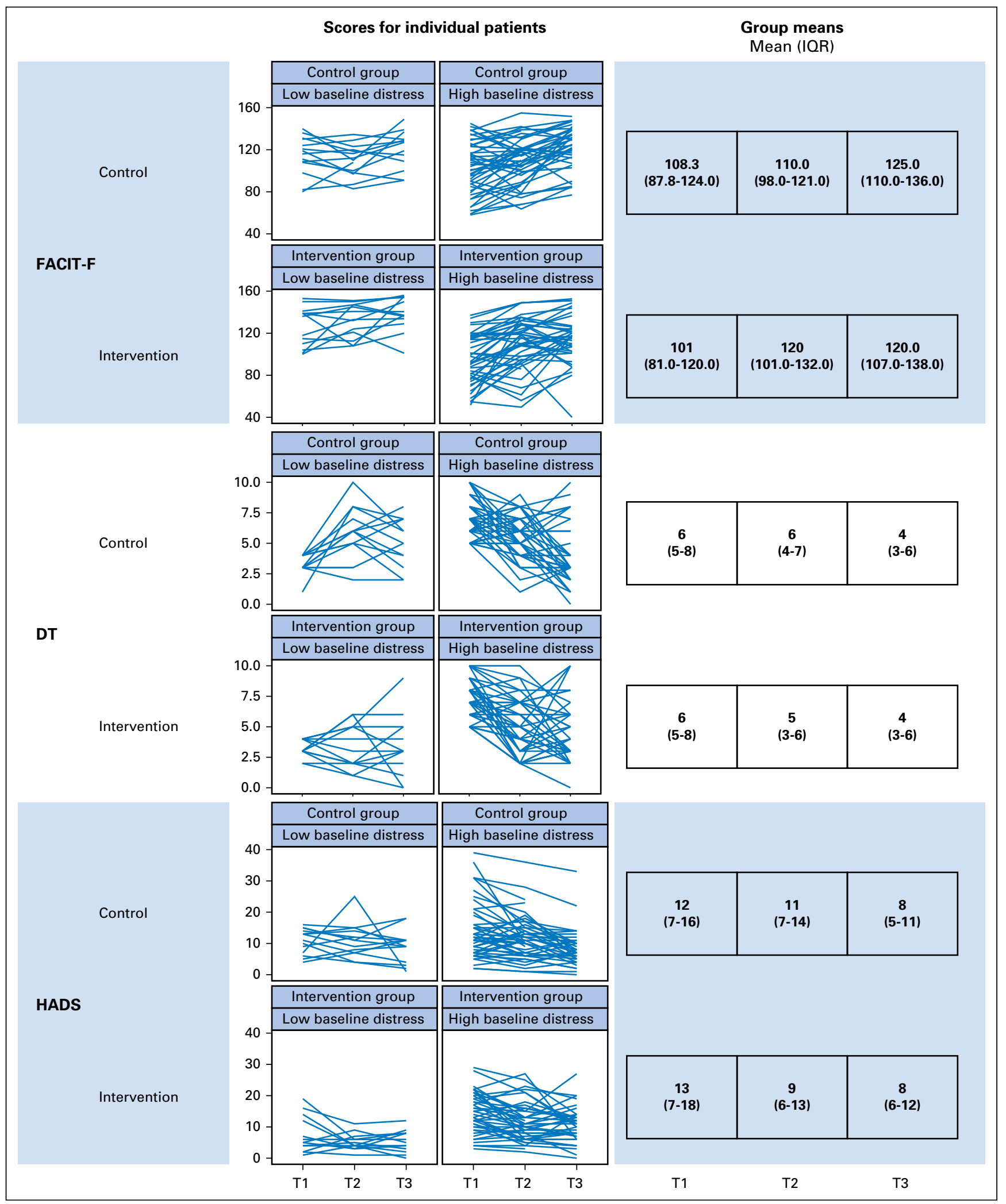

Fig A1. Individual patients' scores and group means for all time points. DT, Distress Thermometer; HADS, Hospital Anxiety and Depression Scale; IQR, interquartile range; FACIT-F, Functional Assessment of Chronic Illness Therapy-Fatigue. 